Bull. Austral. Math. Soc.

$20 \mathrm{c} 07,20 \mathrm{c} 32$

VOL. 41 (1990) [113-115]

\title{
ON THE STRUCTURE OF A REAL CROSSED GROUP ALGEBRA
}

\author{
P.P. PÁLFY
}

\begin{abstract}
We prove that the crossed group algebra $A$ of the infinite dihedral group over the real field defined by the generators $a$ and $b$, relations $b^{-1} a b=a^{-1}, b^{2}=-1$, and $\lambda a=a \lambda, \lambda b=b \lambda$ for all real $\lambda$ is a principal left ideal ring. This corrects a result of Buzási and provides the missing step towards the classification of finitely generated torsion-free $\mathbf{R} G$-modules for groups $G$ which contain an infinite cyclic subgroup of finite index.
\end{abstract}

The aim of this note is to correct Theorem 2.1 from the paper of Buzási with the same title [1]. Professor Buzási had become aware of the error shortly before his sudden death on August 7, 1988. Fate did not let him find the solution.

We will prove that the crossed group algebra $A$ (defined below) is a principal left ideal ring. T'he same methods can be applied to the analogous algebra $B$ over the complex field. These results can be used to complete the classification of finitely generated torsion-free $\mathrm{R} G$-modules for groups $G$ which have an infinite cyclic subgroup of finite index.

This note should be read together with [1]. We will use the same notation and we will also refer to some results from that paper. We deal with the crossed group algebra $A$ of the infinite diliedral group over the real field defined by the generalors $a$ and $b$, relations $b^{-1} a b=a^{-1}, b^{2}=-1, \lambda a=a \lambda$ and $\lambda b=b \lambda$ for all $\lambda \in \mathrm{R}$. We recall some notation from $[\mathbf{1}]$. For $f=f(a)=\sum_{k=m}^{n} \lambda_{k} a^{k} \in \mathbf{R}(a)\left(\lambda_{m} \neq 0 \neq \lambda_{n}\right)$ the norm of $f$ is defined by $|f|=n-m$ and the "conjugate" of $f$ by the formula $\bar{f}=f\left(a^{-1}\right)=\sum_{k=m}^{n} \lambda_{k} a^{-k}$. We have $b f=\bar{f} b$.

TheOREM. A is a principal left ideal ring.

Proof: Let $I$ be a left ideal in $A$. Then $I=I_{1} \cdot d$, where $I_{1}$ is a left ideal generated by some elements $p$ and $1+q b$, where $p, q \in \mathbf{R}(a),(p)=I_{1} \cap \mathbf{R}(a)$ and $d \in \mathbf{R}(a)$ (see [1], Theorem 1.1). By Lemma $1.2, p$ is a symmetric element and $1+q \bar{q} \equiv 0(\bmod p)$,

Received 22 February 1989

Dedicated to the memory of Károly Buzási (1930-1988). Research supported by Hungarian National Foundation for Scientific Research, Grant No. 1813.

Cupyright Clearance Centre, Inc. Serial-fee code: 0004-9729/90 \$A2.00+0.00. 
see (1.5). By definition, $p$ is symmetric if and only if $\bar{p}=\mu a^{-m} p$ for some $\mu \in R$, $m \in \mathbb{Z}$. Obviously $\mu= \pm 1$. We claim that the norm $|p|$ is even and $\mu=1$. Indeed, if $|p|$ is odd and $\mu=1$, then $p(a)$ is divisible by $a+1$; if $|p|$ is odd and $\mu=-1$, then $p(a)$ is divisible by $a-1$; and if $|p|$ is even and $\mu=-1$, then $p(a)$ is divisible by $a^{2}-1$. However, if we consider the homomorphisms $\mathbf{R}(a) \rightarrow \mathbf{R}$ mapping $a$ to 1 or -1 , then $1+q \bar{q}$ is mapped to a nonzero element $1+q(1)^{2}$ or $1+q(-1)^{2}$. Hence $1+q \bar{q}$ can be divisible neither by $a-1$ nor by $a+1$, so the same is true for $p(a)$, hence $|p|$ is even, say $2 n$, and $\mu=1$. Without loss of generality we may assume that

$$
p(a)=\lambda_{n} a^{n}+\lambda_{n-1} a^{n-1}+\ldots+\lambda_{n-1} a^{-(n-1)}+\lambda_{n} a^{-n} \quad\left(\lambda_{n} \neq 0\right) .
$$

Then $\bar{p}=p$.

Let $\nu$ be the natural homomorphism of the group algebra $\mathrm{R}(a)$ onto $\mathrm{R}(a) /(p)$. Further set

$$
W=\left\{\alpha_{n} a^{n}+\ldots+\alpha_{0} \mid \alpha_{0}, \ldots, \alpha_{n} \in \mathbf{R}\right\} .
$$

Obviously, $\nu$ is injective on $W$. Since $(p, q)=1$, it is also injective on $q W$. Now

$$
\operatorname{dim}_{\mathbf{R}} \nu(q W)+\operatorname{dim}_{\mathbf{P}} \nu(W)=2(n+1)>2 n=\operatorname{dim}_{\mathbf{R}} \mathbf{R}(a) /(p),
$$

hence there exist nonzero $w_{1}, w_{2} \in W$ such that

$$
q w_{1} \equiv w_{2} \quad(\bmod p)
$$

We will show that the element

$$
u=w_{1}+w_{2} b
$$

generates the left ideal $I_{1}$. First of all,

$$
u=w_{1}(1+q b)+\left(\left(w_{2}-w_{1} q\right) / p\right) b p \in I_{1}
$$

Since $A$ contains no zero divisors,

$$
N(u)=w_{1} \bar{w}_{1}+w_{2} \bar{w}_{2}=\left(\bar{w}_{1}-w_{2} b\right) u \neq 0 .
$$

Obviously $N(u) \in I_{1} \cap \mathbf{R}(a)$, hence $N(u) \equiv 0(\bmod p)$. On the other hand

$$
|N(u)| \leqslant 2 n=|p|
$$

hence $N(u)=\delta p$ for some nonzero $\delta \in \mathbf{P}$. So we have

$$
p=\delta^{-1} N(u) \in A u
$$


Write $q w_{1}-w_{2}=t p$. Then $1+q \bar{q} \equiv 0(\bmod p)$ gives

$$
\begin{aligned}
w_{1} \bar{w}_{1}(1+q \bar{q}) & =w_{1} \bar{w}_{1}+\left(t p+w_{2}\right) \overline{\left(t p+w_{2}\right)} \\
& =w_{1} \bar{w}_{1}+w_{2} \bar{w}_{2}+p\left(p t \bar{t}+t \bar{w}_{2}+\bar{t} w_{2}\right) \\
& =p\left(\delta+\left(p t+w_{2}\right) \bar{t}+t \bar{w}_{2}\right) \equiv 0\left(\bmod w_{1} p\right)
\end{aligned}
$$

After dividing by $p$ we oblain

$$
\delta+q w_{1} \bar{t}+t \bar{w}_{2} \equiv \delta+t \bar{w}_{2} \equiv 0 \quad\left(\bmod w_{1}\right)
$$

Since $0 \neq \delta \in \mathbf{R}$, we can find $s_{1}, s_{2} \in \mathbf{R}(a)$ such that

$$
s_{1} w_{1}-s_{2} \bar{w}_{2}=1
$$

Then

$$
\left(s_{1}+s_{2} b\right) u=\left(s_{1} w_{1}-s_{2} \bar{w}_{2}\right)+\left(s_{1} w_{2}+s_{2} \bar{w}_{1}\right) b=1+q_{0} b
$$

for some $q_{0} \in \mathbf{R}(a)$. The proof of Lemma 1.5 yields $q_{0} \equiv q(\bmod p)$. Thus

$$
1+q b=\left(s_{1}+s_{2} b\right) u+\left(\left(q-q_{0}\right) / p\right) b p \in A u .
$$

So we have proved that $I_{1}=A u$, hence $I=I_{1} d=A(u d)$ is a principal left ideal.

Similar results can be proved for the analogous algebras over other fields, in particular for the algebra $B$ defined in [1].

\section{REFERENCES}

[1] K. Buzási, 'On the structure of a real crossed group algebra', Bull. Austral. Math. Soc. 38 (1988), 31-40.

Mathematical Institute of the Hungarian Academy of Sciences Budapest, Pf. 127

H-1364 Hungary 